\title{
Editorial: Metabolic Related Cardiomyopathy in Hyperglycemic Patients
}

\author{
Annalisa Capuano ${ }^{1,2}$, Emilio Clementi ${ }^{3}$ and Giuseppe Paolisso ${ }^{4,5 *}$ \\ ${ }^{1}$ Department of Experimental Medicine, University of Campania Luigi Vanvitelli, Naples, Italy, ${ }^{2}$ Campania Regional Centre for \\ Pharmacovigilance and Pharmacoepidemiology, Naples, Italy, ${ }^{3}$ Department of Biomedical and Clinical Science "Luigi Sacco" \\ (DIBIC), University of the Study of Milan, Milan, Italy, ${ }^{4}$ Department of Advanced Medical and Surgical Science, University of \\ Campania Luigi Vanvitelli, Naples, Italy, ${ }^{5}$ Mediterranea Cardiocentro, Naples, Italy
}

Keywords: cardiomyopathy, inflammation, heart failure, mitochondrial dysfunction, IL6

\section{Editorial on the Research Topic}

\section{Metabolic Related Cardiomyopathy in Hyperglycemic Patients}

An appropriate metabolic milieu plays a key role in the physiological functioning of tissues and cells. This is particularly true for cardiac cells, which depend on extracellular glucose and lipid concentrations to contract and transmit electric signals. An appropriate concentration of both extracellular glucose and lipids is required since extremely low or an exaggerated concentration of these metabolic components might have a deranging effect. In particular, extracellular high glucose concentration has been associated with mitochondrial dysfunction and activation of inflammatory pathways, which are frequently associated with abnormal cardiac structure and function and play a pivotal role in the pathogenesis of heart failure (HF) with reduced and preserved ejection fraction.

\section{OPEN ACCESS}

Edited and reviewed by: Ichiro Manabe,

Chiba University, Japan

${ }^{*}$ Correspondence:

Giuseppe Paolisso

giuseppe.paolisso@unicampania.it

Specialty section:

This article was submitted to

Cardiovascular Metabolism,

a section of the journal

Frontiers in Cardiovascular Medicine

Received: 01 December 2021

Accepted: 13 December 2021

Published: 13 January 2022

Citation:

Capuano A, Clementi E and Paolisso G (2022) Editorial: Metabolic

Related Cardiomyopathy in

Hyperglycemic Patients.

Front. Cardiovasc. Med. 8:826914.

doi: 10.3389/fcvm.2021.826914

\section{MITOCHONDRIAL DYSFUNCTION}

The excitation-contraction process consumes a substantial part of ATP produced by mitochondria to support the continuous activity of the heart (1), with a large amount of energy being consumed by the myosin ATPase, SERCAs, and $\mathrm{Na} / \mathrm{K}$ ATPase (2). Mitochondria cover almost $95 \%$ of cardiac cell requests to meet such critical energy requirements through oxidative phosphorylation. Nevertheless, cardiac cells can shift between glucose and lipid oxidation to support a continuous energy output (metabolic flexibility). In normal conditions cardiac cell relies predominantly (almost 60-90\%) on fatty acid oxidation to fuel ATP production while ATP derived from pyruvate oxidation covers the remaining part. Upon increased workload, pyruvate is produced in similar amounts by glycolysis and oxidation of lactate. The relative contribution of the glucose and lipid substrate to ATP production can vary greatly depending on energy request, availability, and hormonal/metabolic milieu. In vitro in the presence of elevated glucose concentration in the culture medium or in vivo in patients affected by type 2 diabetes mellitus morphological and functional mitochondrial changes have been associated with enhanced oxidative stress (3). In particular diabetic cardiomyopathy (DCM) is associated with increased $\mathrm{H}_{2} \mathrm{O}_{2}$ and nitrotyrosine production combined with excessive $\mathrm{Ca}^{2+}$ traffic from the sarcoplasmic reticulum (SR)/endoplasmic reticulum (ER) to the mitochondria, which causes mitochondrial calcium overload followed by an electron transport chain uncoupling and excessive free radicals' production (4). The latter effect might be related to an enhanced SE/ER-mitochondria connection through the over-expression of Mfn2 in the absence of changes in other fusion proteins. In addition, in cardiac cells, specific subpopulations of mitochondria can also be more prone to alterations by the occurrence of abnormal metabolic 
conditions, such as the case of intermyofibrillar mitochondria, typically densely compacted between sarcomeres. The fissionfusion processes can negatively affect their morphology, showing that free radicals imbalance production seems the most damaging factor in DCM.

\section{MYOCARDIAL INFLAMMATION AND DIABETIC CARDIOMYOPATHY}

Myocardial inflammation has been shown to play a critical role in the pathogenesis of $\mathrm{HF}$ with reduced and preserved ejection fraction. Systemic and tissue inflammation is very often associated with abnormal cardiac function and structure (57). From a physio-pathological point of view, hyperinsulinemia, hyperglycemia, hyperlipidemia, and insulin resistance may conspire to the genesis and worsening of DCM leading to HF. In particular, DCM is associated with cardiac hypertrophy and fibrosis with consequent cardiomyocyte cell death. Those tissue events are also a consequence of a pro-inflammatory cascade (5) encompassing the release of TBF-a, IL1b, and IL6 and leukocyte activation, associated with an activation of nuclear factor kappa-light-chain enhancer of activated B cells (NF-kB). This may lead to an auto-potentiating vicious cycle that is activated between cardiomyocytes cell metabolic mediated auto-degeneration and pro-inflammatory mechanisms. Among leukocytes, neutrophils secrete various inflammatory mediators such as cytokines and microparticles and neutrophil extracellular traps (NETs), which aggravates the cardiac injury. In addition, macrophages, classified as pro-inflammatory (M1) and antiinflammatory (M2) cells, lose their balanced ratio with an M1 overactivity. In fact, M1 cells are predominant in type

\section{REFERENCES}

1. Bertero E, Maack C. Metabolic remodeling in heart failure. Nat Rev Cardiol. (2018) 15:457-70. doi: 10.1038/s41569-018-0044-6

2. Stanley WC, Recchia FA, Lopaschuk GD. Myocardial substrate metabolism in the normal and failing heart. Physiol Rev. (2005) 85:1093-29. doi: 10.1152/physrev.00006.2004

3. Lopaschuk GD, Karwi OG, Tian R, Wende AR, Abel ED. Cardiac energy metabolism in heart failure. Circ Res. (2021) 128:1487-513. doi: 10.1161/CIRCRESAHA.121.318241

4. Federico M., De la Fuente S, Palomeque J, Sheu SS. The role of mithocondria in metabolic diseases: a special emphasis on heart dysfunction. J Physiol. (2021) 599:3477-93. doi: 10.1113/JP279376

5. Kaur M, Guan Y, Raja R, Ruiz-Velasco A, Liu W. Mechanisms and therapeutic prospects of diabetic cardiomyopathy through the inflammatory response. Front Physiol. (2021) 12:694864. doi: 10.3389/fphys.2021.694864

6. Paolisso P, Foà A, Bergamaschi L, Donati F, Fabrizio MChiti C, et al. Hyperglycemia, inflammatory response and infarct size in obstructive acute myocardial infarction and MINOCA. Cardiovasc Diabetol. (2021) 20:33. doi: 10.1186/s12933-021-01222-9

7. Paolisso P, Foà A, Bergamaschi L, Angeli F, Fabrizio M, Donati F, et al. Impact of admission hyperglycemia on short and long-term prognosis in acute myocardial infarction: MINOCA versus MIOCA. Cardiovasc Diabetol. (2021) 20:192. doi: 10.1186/s12933-021-01384-6

8. Seferovic PM, Paulus WJ. Clinical diabetic cardiomyopathy: A two-faced disease with restrictive and dilated phenotype. Eur Heart J. (2015) 36:171827. doi: 10.1093/eurheartj/ehv134
2 diabetes, worse insulin resistance by secreting resistin and support DCM progression. T-helper cells (either Th-1 or Th-17) also contribute to DCM since they are associated with increased cardiac hypertrophy, fibrosis, and diastolic abnormalities in diabetic children. Finally, NF-kB activation contributes to myocardial fibrosis, hypertrophy, and ventricular dysfunction and is responsible for a reduced RAAS activation. On the other hand, the advanced glycation end product (AGE) and their receptor (RAGE) triggers $\mathrm{NF}-\mathrm{kB}$ activation, thus aggravating cardiac inflammation.

\section{MYOCARDIAL DYSFUNCTION ON CLINICAL GROUND}

As whole metabolic and immunological cardiac alterations are responsible for impaired cardiac functioning. Such myocardial dysfunction is associated with DCM and HF with preserved ejection fraction $(8,9)$. Indeed, the common soil for both latter diseases is an increased cardiac wall rigidity that does not allow the cardiac chamber to receive enough blood by the bloodstream. Over time, cardiac chambers lose their contractility performance, and a HF at reduced ejection fraction develops. Hyperglycemia has also been shown to negatively affect coronary flow reserve, contributing to the decline in cardiac wall performance or leading to ischemic cardiac insult (8-10).

\section{AUTHOR CONTRIBUTIONS}

AC, EC, and GP contributing to editorial structuring and writing. All authors contributed to the article and approved the submitted version.

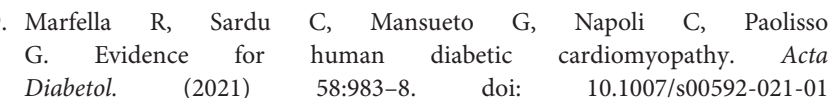
705-x

10. Duque ER, Briasoulis A, Alvarez PA. Heart failure with preserved ejection fraction in the elderly: pathophysiology, diagnostic and therapeutic approach. J Geriatr Cardiol. (2019) 16:421-8. doi: 10.11909/j.issn.1671-5411.2019.0 5.009

Conflict of Interest: The authors declare that the research was conducted in the absence of any commercial or financial relationships that could be construed as a potential conflict of interest.

Publisher's Note: All claims expressed in this article are solely those of the authors and do not necessarily represent those of their affiliated organizations, or those of the publisher, the editors and the reviewers. Any product that may be evaluated in this article, or claim that may be made by its manufacturer, is not guaranteed or endorsed by the publisher.

Copyright $\odot 2022$ Capuano, Clementi and Paolisso. This is an open-access article distributed under the terms of the Creative Commons Attribution License (CC BY). The use, distribution or reproduction in other forums is permitted, provided the original author(s) and the copyright owner(s) are credited and that the original publication in this journal is cited, in accordance with accepted academic practice. No use, distribution or reproduction is permitted which does not comply with these terms. 